\title{
El diseño de las Nuevas Casas Reales de San Luis Potosí Entre lo barroco y lo académico
}

\author{
José Armando Hernández \\ Universidad Autónoma de Zacatecas, México \\ loretoslp@yahoo.com.mx
}

\begin{abstract}
Resumen
Las sedes locales del gobierno virreinal, denominadas Casas Reales, fueron uno de los espacios civiles más importantes en las poblaciones novohispanas. Desde su fundación en 1592, la ciudad de San Luis Potosí no contó con unas Casas Reales dignas; por esta razón, el visitador José de Gálvez determinó en 1767 la construcción de unas Nuevas Casas Reales, acordes con la arquitectura y lustre de la ciudad. No obstante, por cuestiones tanto económicas como de gusto, fueron precisos 23 años para que uno de los diseños presentados fuera aceptado. Es así como, pasando por soluciones castrenses y coqueteos barrocos, y culminando en la sencillez y rigor de la Academia, el edificio y su diseño se circunscriben en la historia como una muestra del cambio de mentalidades, testigo de la transformación que en sustancia y esencia se estaba dando en el siglo XVIII novohispano.
\end{abstract}

Palabras clave: Nueva España, arquitectura, siglo XVIII, Casas Reales, Barroco.

\begin{abstract}
The local government houses of the Viceroyalty, known as Casas Reales, were one of the most important civil spaces in the new world. Since its establishment in 1592, the city of San Luis Potosí did not have a decorous Casa Real, which is why in 1767, the visitor José de Gálvez, determined and decreed the construction of the New Casas Reales, a building in accordance with the architecture and dignity of the city. However, either for economic or style reasons, it took 23 years for one of the three different designs presented, to be approved. And this is how, throughout military solutions, baroque insinuations and concluding with the simplicity and rigor of the Academy, the building and its design circumscribes in history as a sample of the change of mentalities, witness of the transformation that in substance and essence were taken in the New Spain during the 18th century.
\end{abstract}

Key words: New Spain, architecture, 18th century, Casas Reales, Baroque. 


\section{Antecedentes}

Las Casas Reales ${ }^{1}$ primigenias - o Viejas Casas Reales - que tuvo San Luis Potosí se construyeron hacia 1603 (Montejano y Aguiñaga 1972: 12). Dicha tarea había sido propuesta por el alcalde mayor Leonel de Cervantes, sobre el terreno que para tal efecto había dispuesto, en el año fundacional de 1592, el primer alcalde mayor, don Juan de Oñate y Salazar, al hacer la traza de las cuadras y solares del pueblo de acuerdo con las ordenanzas de Felipe II, las cuales indicaban que para que se "pudieran favorecer tanto el Cabildo, como la Aduana, Ataranzas y Templo", estos se hicieran contiguos (11). El lugar elegido fue el costado nororiental de la plaza principal, aledaño a la parroquia. La construcción primigenia era de una sola planta, con portales de ocho arcos y techos de tejamanil, y fue concluida hacia 1606 (12). Esta construcción dejó mucho que desear, ya que constantemente había que estarle haciendo composturas y adecuaciones.

Antes de terminar la primera mitad del siglo XVII la bonanza minera que había convertido a San Luis Potosí en un destino de interés tanto para mineros como para comerciantes empezó a decrecer; no obstante, el pueblo recibió el título de ciudad el 30 de mayo de 1656, por orden del Virrey Duque de Alburquerque, lo cual fue confirmado por el rey Felipe IV el 17 de agosto de 1658. La concesión de esta nueva categoría no mejoró las Casas Reales (Montejano y Aguiñaga 1972: 13), las cuales siguieron en franco deterioro hasta el año de 1743, en que las Antiguas Casas Reales tomaron su forma definitiva y se mantuvieron más o menos presentables durante los años siguientes.

El año 1767 fue decisivo en la historia del pueblo potosino y, en general, de la Nueva España; una serie de acciones subversivas en contra del gobierno virreinal, conocidas como tumultos, coincidentes con levantamientos acontecidos en Apatzingán, Uruapan, Pátzcuaro, Guanajuato, San Luis de la Paz y San Felipe (Monroy Castillo y Calvillo Unna 114), tuvieron lugar en la ciudad de San Luis Potosí desde el 10 de mayo hasta el 24 de julio. En el marco de estos levantamien-

Toda fundación en la América hispana durante la dominación española contemplaba, desde su gestión, la construcción de un edificio que fuera sede del gobierno virreinal, donde se representaran los intereses del rey, se impartiera justicia y se verificara la vida política de la población o provincia. A estas construcciones se les denominaba Casas Reales (en algunos casos, también, Casas del Rey), las cuales eran utilizadas como casa de habitación de los oficiales reales y sede del cabildo, así como armería, y, en muchas ocasiones, cárcel, como fue en el caso de San Luis Potosí desde el principio de su fundación y en los dos proyectos de Casas Reales que se construyeron en la ciudad. En algunos casos, la funcionalidad del edificio como sede gubernamental trascendió el período virreinal, y estas se convirtieron, por continuación, en las sedes de los gobiernos independientes; el caso de las Nuevas Casas Reales potosinas es prueba de ello, al convertirse en Casas Consistoriales al inicio del período independiente de México y, posteriormente, en Palacio de Gobierno de la actual entidad federativa de San Luis Potosí. 
tos la plaza principal de la ciudad sufrió muchos daños; en especial, las de por sí paupérrimas Casas Reales. Esto condujo a que el visitador José de Gálvez, quien había llegado el 24 de junio para someter a los sediciosos, dispusiera una serie de acciones para la mejora y decoro de este vital espacio arquitectónico, sede de los representantes del gobierno virreinal.

En cartas fechadas el 10 y el 12 de octubre, José de Gálvez, quien se encontraba a punto de salir de San Luis Potosí rumbo a Guanajuato (Gálvez 60), dejó indicado por escrito al Ayuntamiento las acciones que respecto al tema de las Casas Reales había dispuesto. Estas indicaciones mencionaban la necesidad de construir con la mayor brevedad posible unas Nuevas Casas Reales y cárcel, debido a que, en el trance de los tumultos, el edificio que servía para tal fin había quedado en una "deplorable situación" (Montejano y Aguiñaga 1973: 29), a causa de "los rebeldes en sus repetidas invasiones contra los jueces y principales vecinos de San Luis" (Gálvez 57). En su decreto no solo dejó esta disposición, sino que en el mismo daba la indicación exacta y precisa acerca de la ubicación, el acabado y el encargado que debería hacerse con la tarea de construirlas. El propio Gálvez lo confirmó en el informe que rindió al Virrey Marqués de Croix el 25 de diciembre de 1767, en el cual mencionó la necesidad de dejar a la ciudad en algún régimen y en más lustre del que tenía (58), e hizo alusión al hecho de que las instalaciones donde se desempeñaban los cargos gubernamentales no iban de acuerdo con la dignidad de sus representantes. El informe aclaraba: “...carecía el público de un magistrado competente [...] y la policía que debe haber en una población tan recomendable como lo es la de San Luis Potosí [...], [la cual] se halla dichosamente situada en uno de los más hermosos, fértiles y templados valles de este reino y sus edificios públicos y particulares son de una arquitectura bastantemente exquisita y suntuosa para la América" (58).

Al ser la arquitectura potosina - tal como fue descrita - "exquisita y suntuosa", resultaba imperativo, desde su visión, elevar a tal grado de distinción y categoría al edificio más importante de la vida política y pública potosina. En teoría, el proyecto que planteaba Gálvez resultaba de lo más atractivo; sin embargo, la bonanza minera había decrecido hacía mucho tiempo ya, y una construcción de esta magnitud resultaría de lo más costosa. Previendo esto, Gálvez dejó instrucciones para esto tanto en el ámbito de la mano de obra como en lo económico: el decreto precisaba que el coste "corriera a expensas, como corresponde, del bajo vulgo que causó los daños" (57), por lo que los jornaleros y los naturales de los barrios trabajarían en cuadrillas alternadas en la construcción, y se les pagaría únicamente lo necesario para su manutención; y finalmente, para hacerse con recursos, se instruía el cobro de un impuesto consistente en una tasa de dos reales por cada fanega de maíz vendida. 
Tal disposición no fructificó, y tan solo a un año y dos meses de haberla dejado Gálvez el pueblo potosino argumentó, a manera de oposición, que el impuesto era excesivo e insostenible, y que el coste de una obra de esta magnitud era tal que resultaba imposible llevarla a cabo. Los argumentos eran sólidos y, en efecto, no era posible en ese momento solventar un gasto de ese tipo. En palabras del propio Andrés de Urbina, alcalde mayor de la ciudad, quien llevó un ocurso al Virrey (fechado el 23 de diciembre de 1768), las medidas tomadas por Gálvez habían sido factibles en aquellas circunstancias, pero, dadas las condiciones económicas que vivía la ciudad, estas resultaban inalcanzables, principalmente porque las minas estaban "tan extenuadas que se necesitan correspondientes arbitrios para restablecerlas" (AHESLP f. 120r-129r); estas se convirtieron en razones suficientes para postergar la recaudación de impuestos y posponer la construcción del recinto.

El proyecto de las Nuevas Casas Reales se quedaría solo en eso durante los 20 años siguientes, y aunque se seguían cobrando los arbitrios impuestos al maíz y el descontento se generalizaba por esta disposición (AHESLP f. 202r-206r) ${ }^{2}$, el proyecto de construir el nuevo recinto de gobierno no avanzaba. Fue solo hasta el cambio a una administración basada en intendencias, y con la llegada del primer intendente de San Luis Potosí, Bruno Díaz de Salcedo, cuando el proyecto tomó, de nueva cuenta, importancia.

El 3 de septiembre de 1787 se abría una carta en la Sala Capitular del Cabildo potosino, en la cual Bruno Díaz de Salcedo comunicaba su empleo como Intendente (AHESLP f. 21v-22r). Una vez en actividades de gobierno, y no sin cierta improvisación en materia de espacio, quedó de manifiesto la urgencia de concretar el proyecto que había dejado Gálvez 20 años atrás: "Capitular de San Luis Potosí y noviembre 17 de 1787. Juntos en ella los señores Presidente Don Bruno Díaz de Salcedo, y demás capitulares de este Ilustre Ayuntamiento en la casa habitación del señor Presidente por indecencia de las Casas Reales" (AHESLP f. 42v).

El lustre del que hablaba Gálvez en su orden de 1767 seguía faltándole a la sede del gobierno potosino, ahora, además, sede de la Intendencia más grande de Nueva España; por ello, pocos días después, y de nuevo en la casa habitación del presidente, se dictaba: "En consideración a lo indecente y deteriorado que se hallan las Casas Reales y amenazando ruina se acordó se celebren por ahora y hasta tanto que se construyen las Casas Reales los acuerdos o cabildos en la posada del Señor Presidente" (AHESLP f. 51r-51v).

2 Aunque el arbitrio aplicaba únicamente si la fanega de maíz alcanzaba los 12 pesos, cosa que era poco común, por lo cual la obra se iba postergando por falta de recursos. 
A la realidad de que la sede del gobierno no presentaba las condiciones adecuadas para llevar a cabo la actividad para la cual estaba destinada, habría que agregarle la visión absolutista del nuevo representante del gobierno virreinal. El hecho de que la figura de Intendente comprendiera todos los ámbitos del gobierno - el militar en primera instancia - en una sola persona, aunado a la visión de los borbones, para quienes el Estado tenía una función totalitaria, controladora y omnipresente - el cual funcionaría en la medida en que los ciudadanos funcionaran-, terminarían por constituirse en los factores tanto físicos como ideológicos que precipitarían la construcción de las Nuevas Casas Reales; finalmente, un gobierno no podría inspirar respeto e imposición algunos (lo cual se buscaba desde los levantamientos de 1767) cuando la sede desde la cual representaban a la Corona no tenía la dignidad suficiente para albergar a dichos representantes. Habría que buscar de una vez por todas que se iniciara un proyecto tan largamente postergado.

\section{Las Nuevas Casas Reales y sus protagonistas. Un proyecto entre el Barroco y la Academia}

Todo proyecto constructivo implica la existencia de un diseño previo. Se contaba ya con la superficie sobre las que se desplantarían las Nuevas Casas Reales, la cual estaba señalada desde 1767. Faltaba tan solo proponer un proyecto para ejecutarse, el cual contaría con varios autores, y cuyas propuestas verían la transición entre el barroco y la Academia.

\section{José de Gálvez Gallardo}

En un estricto sentido de justicia, podemos afirmar que el primer proyecto y diseño de las Nuevas Casas Reales no proviene de un maestro arquitecto, sino que se le debe al propio José de Gálvez. Al parecer, el visitador no se contentó con solo dejar las instrucciones para que se construyeran las Casas Reales: además, ordenó que se hicieran los planos respectivos. En una carta fechada el 10 de octubre de 1767, dictada por Gálvez a Prudencio Ochoa Badiola, quien la signa, podemos intuir la formación de un plano que proyectaba el edificio pensado por este "desde su arribo", lo cual quiere decir que en los meses de julio y agosto determinó el visitador la ejecución de este edificio; mas, hombre prevenido, posiblemente dejó los planos — al menos los mandó realizar — para que se ejecutase su Real Orden. La carta así lo manifiesta:

Para prevenir a la indispensable y ejecutiva urgencia en que las anteriores rebeliones, y los ningunos fondos públicos de propios y Arbitrios han constituido a esta Ilustre Ciudad, dejándola sin Casas Reales, ni cárceles seguras, determiné desde mi arribo que en el cuadro que hace frente sobre la Plaza a la 
Iglesia Parroquial se fabriquen unidas dichas Casas Reales, la de la caja de su Majestad y la Cárcel pública, con todas las oficinas correspondientes a cada uno de estos Edificios tan indispensables como útiles a la seguridad, decoro y ornato de esta importante población. Y supuesto que habiendo dado cuenta de mi determinación al Excmo. Sr. Marqués de Croix, Virrey y Capitán General de este Reino, se sirvió S.E. aprobarla con el plan que por mi orden se formó de la obra. (YUL, f. 21r-22v)

En la carta Gálvez habla de un proyecto aprobado, cuyo "plan se formó"3 en el entendido de que debería cubrir ciertas características, las cuales se detallan en el acta de Cabildo de 1768, en la cual se da testimonio de la orden dada por el visitador para que Felipe Cleere se hiciera cargo de la construcción, mas no del diseño, por lo cual concluimos que este ya existía en la forma del plano que mandó realizar entre julio y agosto:

... he determinado que se construya una nueva fábrica en la cual se comprendan igualmente así las Casas Reales y cárceles referidas, como la caja con todas sus oficinas, las tres viviendas de los dos oficiales Reales y el ensayador, para que luego que se conduzcan las platas a la Real Fundición y ensaye, se evite todo riesgo a los particulares dueños, y por consiguiente, a los Reales intereses; cuya sala deberá bien comprender una sala de armas para custodia de las que tenga la ciudad, de las pertenecientes a las Milicias Provinciales, con su vestuario; asimismo las piezas que alrededor de ella y en su primer piso, puedan estrechamente acomodarse, cuyos arrendamientos quedarán al beneficio de la ciudad. Para verificar el todo, he resuelto que se ejecute en el cuadro entero, que ocupan las casas de todo el frente de la Plaza que mira al Oriente y la parroquia y que contiene la Alhóndiga y demás habitaciones de los lados y espaldas del cuadro inmediato a la Real Caja que hoy existe, con cuatro pequeños baluartes en los cuatro ángulos, en donde se coloque un proporcionado número de pequeños Cajones de Artillería, o Terreros, que servirán de defensa y oposición a cualquier motín o sedición que intente la plebe en lo sucesivo, como últimamente se ha experimentado con insolente desobediencia a las Leyes y determinaciones de Nuestro Soberano, temeraria irreverencia a los Jueces. (AHESLP f. 118r-119r)

3 En el léxico de la época, mandar formar un plan, o plano o planes, equivalía a mandar a hacer los dibujos que servirían como guía de la obra. Nótese en los párrafos siguientes cómo, al referirse al diseño de los edificios y de la hechura de sus planos, se describe el acto como mandar a formar los planes o planos o plan. Véase la nota 36, acerca de la petición que hace el Virrey de que se le entregue un Plan y presupuesto formado por peritos de la obra proyectada y mandada construir por el excelentísimo Sor. Marques de Sonora... 
Las instrucciones dadas por Gálvez nos permiten intuir cuál era el concepto y el diseño que buscaba que cumpliera el edificio, sobre todo, en materia de espacio, ya que anticipa la necesidad de dos pisos - las Antiguas Casas Reales sólo tenían uno- (Montejano y Aguiñaga 1972: 12), la necesidad de habitaciones específicas y la necesidad de que en torno al primer piso se construyeran piezas que serían arrendadas. Gálvez pensaba — y en el diseño posiblemente así estaba formulado- en un espacio típico con arcadas, en el que se contemplaban espacios para comercio así como para el resguardo de armas. Muy interesante resulta que, desde su perspectiva de visitador y ejecutor de ley, anticipa la necesidad de ubicar cuatro baluartes con sus respectivos cajones de artillería en las esquinas del recinto, con lo cual cubría en su diseño la necesidad defensiva que tenía este edificio. José de Gálvez no era un simple jurista; dentro de su formación y discurso podemos ver la clara visión ilustrada de su época, por lo que no resultaba extraño que conociera de diversos temas, como lo era el del arte de la fortificación, tal como lo demuestra el hecho de que entre los libros que trajo de su biblioteca personal de España a México se encontraban los 14 tomos del Compendio Mathematico (Madrid, 1712), de Tomás Vicente Tosca, padre de la orden filipense (Rubio Mañé 36), cuyo tomo número $\mathrm{V}$ versa específicamente sobre el arte de la arquitectura civil y militar, y sobre la pirotecnia. Dicho tratado ejemplificaba y recomendaba ciertos elementos arquitectónicos, con la finalidad de brindar mayor seguridad a los moradores de los edificios; entre ellos, el uso de baluartes defensivos ${ }^{4}$, tal como sugirió Gálvez que se utilizaren en su proyecto de las Nuevas Casas Reales. El proyecto confirmaba la necesidad de un espacio digno, al tiempo que daba solución inmediata a una problemática que se había presentado, y que, en la visión del visitador, bien podía pasar de nuevo y prontamente: "que servirán de defensa [los baluartes y artillería] y oposición a cualquier motín o sedición que intente la plebe en lo sucesivo, como últimamente se ha experimentado con insolente desobediencia a las Leyes y determinaciones de Nuestro Soberano" (AHESLP f. 118r-119r).

Estas disposiciones acusan el pragmatismo claro y eficiente de Gálvez respecto a necesidades y problemáticas tan diversas, pero tan caras al Reino, y no podemos sino pensar que dentro de este pragmatismo, a la par de la orden dada para la construcción del edificio, dispuestos los arbitrios para su consecución y nombrado el encargado de la construcción, existieran también los planos para su ejecución. A pesar de que no tenemos noticia — más allá de lo aquí expuestosobre la ejecución de los planos ordenados por Gálvez, amén de que la persona comisionada para ejecutar la construcción del edificio, conforme con lo dispuesto

$4 \quad$ La definición de baluarte que se da en el tratado del padre Tosca es la siguiente: "Baluarte, es un cuerpo pentágono, que puesto en los ángulos de la plaza, sale abanzado azia la campaña para defender el muro" (258). 
por el visitador, se negó a hacerlo, y de que el Cabildo dispuso al poco tiempo que era mejor y más barato reedificar las Antiguas Casas Reales (YUL f. 36v), no se puede olvidar que el primer diseño que tuvieron las Nuevas Casas Reales fue formulado y dispuesto por José de Gálvez Gallardo.

\section{Francisco Bruno de Ureña}

Tal parece que en el momento en que Felipe Cleere rechazó el cargo conferido por don José de Gálvez, y que el Cabildo decidió — aunque nunca lo hiciere - reedificar las Antiguas Casas Reales, toda posibilidad de diseño de un nuevo edificio, y con ella su construcción, se cerró también. Pasarían 21 años para que el proyecto fuera retomado, ahora sí, por un maestro en arquitectura: Francisco Bruno de Ureña. El primer dato que tenemos de él es que era hijo de Felipe de Ureña, quien tuvo su taller de escultura y ensamble en la Ciudad de México durante el primer tercio del siglo XVIII, y que influiría de forma determinante en la introducción de la pilastra estípite en el centro y norte de la Nueva España. Quizá motivado por su padre, quien tenía un taller de construcción en Guanajuato (Reséndiz García 23-28), Francisco Bruno de Ureña tomaría el camino de la arquitectura, que lo llevó a obtener el título de maestro:

El excelentísimo señor Virrey de este Reino, se ha servido nombrar a don Francisco Bruno de Ureña, vecino de la ciudad de Guanajuato, por Maestro de Arquitectura: Perito medidor de Tierras y Aguas en toda esta Nueva España, notifícolo a V. S. por lo que toca al Real Derecho de Media Annata, de cuyo entero previamente se tomará razón en el Tribunal y Real Audiencia de Cuentas. México, 10 de octubre de 1776. ${ }^{5}$ (AGN Media Annata, vol. 181, f. 410r.).

Entre 1776 y 1789 Bruno de Ureña desempeñó muchas actividades: a él se le debe la traza del retablo barroco de San Nicolás Tolentino, de la parroquia del Real de Minas de Guanajuato, el cual terminó el maestro Francisco Berro (Reséndiz García 26-27), ya que a Ureña se le encargó hacer un levantamiento y planos para un proyecto de ciudad en Catorce, San Luis Potosí. El proyecto en cuestión le fue encargado por don Silvestre López Portillo para que realizara la traza completa de una nueva población que estuviera a dos leguas de las minas de Catorce, cuya justificación era la de cambiar el Real formado espontáneamente cerro arriba, junto a las minas, a un lugar adecuado por su topografía (Montejano y Aguiñaga 1975: 2728). El 16 de marzo de 1780 Ureña presentaba con los siguientes títulos su proyecto:

$\overline{5}$ Nombramiento por el cual pagó la cantidad de 27 pesos 5 tomines, el 11 de octubre de 1776. 
Mapa hinografico, y desplante horizontal que yo don Francisco Bruno de Ureña, Agrimensor titulado por S.M. (q.D.g.) de tierras, Aguas y Minas para todo el Reino de Nueva España, maestro examinado por el S.G. en los Artes de Arquitectura, Maquinaria, Montea, y cantería y en todo lo conducente a estas facultades, hice por orden del señor Comisionado don Silvestre López Portillo para la distribución del terreno en Plazas, Cuadras y calles de la Nueva Población de Nuestra Señora de Guadalupe. (Velázquez 392)

Desafortunadamente, tanto para Silvestre López como para Bruno de Ureña, y a pesar de la prudencia del proyecto, este nunca se llevó a cabo, por el aferramiento de los pobladores del Real de Catorce hacia sus minas (Montejano y Aguiñaga 1975: 29). Este proyecto fallido tendría un desenlace aún más polémico entre sus promotores: el 10 de julio de 1784 Silvestre López firmaba una carta dirigida al Virrey, en la cual se declaraba inocente de las acusaciones que en su contra hacía Bruno de Ureña respecto a un testimonio de posesión de la mina de Zavala, la cual reclamaba para sí, al igual que el bachiller Manuel Flores (Montejano y Aguiñaga 1975: 7-12) .

Entre 1786 y 1792 se realizaron los retablos en piedra de San Juan de la Cruz y del Señor de los Afligidos, del templo del Carmen de San Luis Potosí (Martínez Rosales 293), los cuales conservan en sus calles laterales sendas pilastras estípites de complicada forma, lo cual nos hace intuir la presencia del maestro Ureña haciendo gala del legado escultórico de Felipe, su padre, y rindiendo un último tributo al estípite, próximo a desaparecer. Aunque no queda claro en qué momento Ureña fue considerado para el diseño de las Nuevas Casas Reales, este seguramente se le solicitó mientras realizaba dichos retablos.

Infortunadamente para Ureña, su proyecto, al igual que el de la Nueva Población de Nuestra Señora de Guadalupe de los Catorce, se vería rechazado, ya no por la terquedad de unos mineros, sino porque habiendo nacido barroco, el proyecto se enfrentaba ahora al fervor ilustrado de la época. El 10 de marzo de 1790 el intendente Bruno Díaz de Salcedo enviaba una carta al cabildo de la ciudad, en la cual exponía la petición hecha por el Virrey, desde el 24 de agosto de 1789, quien insistía en que se entregara el proyecto para las Nuevas Casas Reales:

Con fecha de 6 del corriente mes me ordena el Excelentísimo Señor Virrey, previo pedimento del señor Fiscal de Real Hacienda, que dirija a S.E. un Plan y presupuesto formado por peritos de la obra proyectada y mandada construir por el excelentísimo Señor Marqués de Sonora [...], con la correspondiente

\footnotetext{
$\overline{6}$ El original de la carta se encuentra en AGN (Minas, vol. 112, 1784, s. fol).
} 
regulación de su costo: y estando formado el plan del dicho edificio, debiendo quedar aquí una copia de él para que sirva de dirección en la construcción, prevengo a $\mathrm{V}$. S. disponga que se saque del mismo original una copia con la regulación de lo que pueda costar, y del tiempo que se necesita para su conclusión, informándome al mismo tiempo que se me dirija este documento de cuanto ocurra y considere V. S. digno en el asunto.

Urge el cumplimiento de dicha orden para darlo al expediente promovido en la materia $[\ldots]$.

S. L. Potosí, 10 de marzo de 1790. Bruno Díaz de Salcedo

AHESLP Ayuntamiento, 1790, f. 36r-36v)

El Cabildo de la ciudad contestó que se sacaría con la mayor brevedad una copia del "plano formado" (AHESLP Ayuntamiento, 1790, f. 40v), lo cual nos permite inferir que ya existían los planos y que el proyecto ya se había mandado a hacer con anterioridad. Pero a pesar de la premura, el Cabildo se dilataría dos meses más en dar cauce a esta petición, e informaría lo siguiente:

...pasa este Cabildo por éste, a satisfacer a V.S. en el todo, su segundo dicho oficio, y dice que presenta a V.S. dos Planes, que en el uno se demuestra la planta horizontal de la obra proyectada de Casas Reales y Cárcel, así en los bajos, como en sus altos, y otro que demuestra la fachada del edificio: quedando como queda en poder del Cabildo, para la dirección de la obra, otros dos tantos, según, y como los que presenta a V.S.; y todos los dichos Planes van firmados del perito que los dispuso, e hizo, que fue el Maestro examinado de Arquitectura, ahora ya difunto, don Bruno Ureña, quien con otro perito hizo regulación, de que la obra pudiera tener de costo hasta 40 ó 44 mil pesos; y aunque primero se había hecho otro juicio más subido, pero después habiéndose instruido el otro perito, en lo barato de materiales y operarios que se consigue en esta ciudad, se hizo el mismo juicio, que economizó más el compañero por la experiencia que tiene, como originario vecino y práctico en esta ciudad. Y prosiguiendo a contestar este referido segundo oficio: se hace juicio el Cabildo, como también se ha hecho el mismo, el práctico que vive, que puede tardar la obra en su construcción de cinco a seis años, vistas las circunstancias de reales, tiempo y demás.

Sala capitular de San Luis Potosí, y Mayo 14 de 1790

(AHESLP Ayuntamiento, 1790, f. 79v) 
Quedaba claro, con este oficio, un par de cosas: la primera, que el proyecto de Ureña era de $1789^{7}$ y no de 1790 , como se ha referido (Montejano y Aguiñaga 1973: 44), y que dicho maestro había muerto también por aquel año. La carta explica que los planos estaban conformados por dos dibujos: uno de la planta del edificio, en el cual se proyectaban dos niveles, y uno de la fachada, del cual se infirieron las vistas del patio o patios interiores. Más importante fue que en el proyecto administrativo participó un alarife potosino, que conocía bien de la actividad constructiva de la región. Se escapa el nombre de este personaje; sin embargo, y por como se dieron las circunstancias en torno al proceso constructivo, podemos intuir que el perito del que se habla pudo ser el maestro en arquitectura José Joaquín Martínez, quien a la postre se convertiría en director de la obra de Nuevas Casas Reales. Desconocemos qué fue del par de juegos de planos que se sacaron; sólo se sabe que uno de ellos llegó a manos del director de arquitectura de la Academia de San Carlos, Antonio González Velázquez, por cuyo conducto se sabe que la propuesta de Ureña conservaba la esencia del barroco, razón suficiente para que la Academia no permitiera que este se ejecutara.

\section{La Academia}

La Academia, como institución reguladora de las artes, aparece también bajo el mandato de la Casa de Borbón. Su formación se debe, en parte, al esfuerzo que hizo el Estado español por ponerse a la altura del resto de Europa en cuestión de arquitectura principalmente; y "ponerse a la altura" consistía en erradicar todo vestigio de esencia barroca reemplazándolo por una nueva, ilustrada y acorde con el momento; así, nace en 1752 la Real Academia de San Fernando en Madrid, mandada a fundar por el rey Fernando VI (Rodríguez G. de Ceballos 18, 41). En México la creación de la Academia se debe a que en 1778 el rey Carlos III le encomendó al grabador Jerónimo Antonio Gil la tarea de establecer una escuela de grabado en Nueva España, que sería precursora de la Academia, la cual terminaría abriendo sus puertas solemnemente en 1785 (Fuentes Rojas 14).

En San Luis Potosí la noticia de la fundación de la Academia de San Carlos venía acompañada en un principio por la petición de dinero para su constitución (AAASC doc. 10023). En un oficio firmado por Lorenzo Montoya, del Cabildo de la ciudad, fechado el 6 de diciembre de 1785, año en el que propiamente abrió sus puertas la Academia, se daba constancia de la recepción de los "estatutos e instrucciones de la Real Academia", los cuales Montoya se comprometía a pro-

En las actas de Cabildo de la ciudad de 1788 no se menciona nada acerca de la comisión para formar los planos de Casas Reales. Desafortunadamente, las actas relativas al año 1789 no existen en el Archivo Histórico de San Luis Potosí, por lo que no podemos comprobar esta última aseveración más que con el oficio citado. 
mulgar a la mayor brevedad (AAASC Gaveta 2, expediente 120). Algunos días después, el 26 de diciembre, el Ayuntamiento de San Luis Potosí emitía una carta dando cuenta de la recepción de la "orden de erección, fundación y dotación de la Real Academia de San Carlos" (AAASC Gaveta 2, expediente 73), con lo que quedaba debidamente establecida y en inteligencia tanto del Cabildo como de la población en general.

La fundación de la Academia le daría a la arquitectura un nuevo cariz, por lo que la organización de la tarea constructiva — tan cara a las ideas ilustradas-implicaba nuevos mecanismos de control, regulación y censura. La Academia sería, pues, el aparato intervensionista que normaría el quehacer arquitectónico desde su inicio como proyecto, hasta la censura en la tarea de edificación. Emulando a la Academia de San Fernando 8 , la Academia de San Carlos de la Nueva España se encargaría de criticar la arquitectura y, también, de autorizar las construcciones en la ciudad de México en función del análisis de sus planos (Fuentes Rojas 26).

Para el caso del interior de la Nueva España, el asunto era un poco diferente: los cabildos podían hacer nombramiento y dar título de director de fábrica o de alarife de la ciudad ${ }^{9}$, por lo que la intervención de la Academia en estos territorios era menor, tanto por la lejanía como por la falta de académicos que pudieran regular la producción en concordancia con los preceptos y estatutos de la propia Academia (Fuentes Rojas 26). Estas salvedades habrían podido permitir que el proyecto de Ureña, con todo y haberse concebido como barroco, se materializara. Desafortunadamente para su causa, la urgencia de construir este recinto tenía, al momento en que se presentaron sus planos, 23 años, y dado que en los estatutos e instrucciones de la Academia de San Carlos se establecía que todos los planos que se hicieran debían ser revisados y aprobados por el director de arquitectura, aquellos formados por Ureña fueron remitidos a Antonio González Velázquez (AAASC, Gaveta 4, expediente 581, carta 1) ${ }^{10}$. El dictamen de este fue categórico:

$8 \quad$ En 1777 el rey Carlos III decretó, mediante reales órdenes, que ningún magistrado de los Ayuntamientos ni entidad eclesiástica iniciara obra alguna sin que su proyecto fuera antes supervisado y, en su caso, corregido por la Academia; años después, en 1786, se formaría en la Academia de San Fernando una comisión de arquitectura, la cual tenía por objetivo examinar todos los planos, monteas y perfiles presentados; eliminar los excesos decorativos o rechazarlos, y formar unos nuevos y adecuados al gusto ilustrado (Rodríguez G. de Ceballos 20).

9 Como sucedería con el ya mencionado José Joaquín Martínez, quien recibió de parte del Cabildo el título de Maestro Mayor de Alarife de la Ciudad, el 6 de febrero de 1793 (AHESLP Ayuntamiento, 1793, f. 21v.).

10 Cfr. Montejano y Aguiñaga (1973: 45). 
México 16 de agosto de 1790 [...] Señor Presidente de la Real Academia de San Carlos de la Nueva España. Los planos y elevaciones, ejecutados por don Francisco Bruno de Ureña, que en oficio de 18 de agosto me ha remitido V. S. para que informe sobre su arreglo y disposición, digo que me es bien sensible el dar mi dictamen en una materia a donde, si ha de ser conforme a mi instituto, temo ser sindicado de acre y duro al parecer de los mismos que yo del buen gusto de esta Arte.

Pues dichos diseños están llenos de errores y defectos de suerte que prometiendo en la explicación orden jónico, demuestra un desconcertado conjunto en su delineación, al que no se puede dar nombre propio. Las basas demuestran ser un mal formado toscano, y los capiteles de la misma suerte, corintio. Las cornisas y una muchedumbre de inútiles molduras que no hacen relación alguna con el edificio, llenas de un cúmulo de cantones de gusto extravagante de churriguera, no hallándose cosa alguna de la orden que promete.

Los arcos del patio principal, en la parte baja debiendo tener de alto el duplo de su ancho, tienen poco más de su cuadrado; cuya mala proporción obliga a los de las altas a la ridícula figura que gozan; que además de ser desagradable a la vista, son de la peor construcción y menos subsistentes: y aunque no faltan autores que describen semejantes arcos, no deben hacer ley, pues en todas Artes y Ciencias se hallan ridiculeces dignas de mayor desprecio, escritas en siglos menos Ilustrados que el presente.

En cuya virtud los alzados son dignos de total reprobación, pues después de lo dicho, es gasto inútil. Las plantas pueden tolerarse, aunque a poco que se moviere en ellas se podrían formar unos alzados bien arreglados de Arquitectura de buen gusto.

Por último, sin embargo de los títulos con que se condecora dicho Francisco Bruno de Ureña, me parece de conveniente insinuar a V. S. que le considero comprendido en el Artículo 29, folio 64, N 3 de los estatutos: es cuanto a cumplimiento de mi obligación debo decir a V.S. sobre el particular. México, y agosto 18 de $1790^{11}$ (AAASC, Gaveta 4, expediente 581, carta 2)

Los planos no solo fueron rechazados, sino que, además, se sugería tomar la decisión de prohibirle a Ureña dirigir obras (Fuentes Rojas 27). No era la primera vez que Velázquez hacía un dictamen tan desfavorable respecto al trabajo de los arquitectos de la vieja escuela: incluso sin conocimiento previo, había criticado obras que, por barrocas, él consideraba deformes y peligrosas. Acaso el ejemplo más claro de su total aversión hacia lo que no fuera académico fue la crítica infundada que hizo en 1798 acerca de la sede de la Inquisición en la ciudad de México.

$11 \quad C f r$. Montejano y Aguiñaga (1973: 45-46). Existen algunas diferencias en nuestra transcripción del original respecto a la hecha por el padre Montejano. 
La crítica de González Velázquez se centraba en la disposición de los arcos, los cuales presentaban en las esquinas una voladura a manera de pinjante, en vez de bajar con una columna, y a los que atribuyó el apelativo de "ridículos" y "peligrosos" (Santos Zertuche 156-157). Presto, el arquitecto se dispuso a formar unos planos que contemplaban construir de nuevo los arcos "que estaban en el aire" o, en su defecto, cambiar la planta del patio por una ochavada. La Inquisición, con su habitual discreción, tomaría una segunda opinión: la del sobrestante José Antonio Zúñiga, el cual trabajaba para el tribunal; éste diría que lo que en apariencia eran cuatro arcos con una de sus columnas al aire eran en realidad dos, y que todos tenían su empuje tectónico desde la pared, por lo que el pinjante que quedaba volando únicamente estaba haciendo las veces de aparentar que se trataba de cuatro arcos, y que, finalmente, lo único que se requería era algún remozamiento en el acomodo de las piedras (Santos Zertuche 157). No era esta la primera vez que Velázquez criticaba unos "arcos al aire", pues en 1796, con motivo de revisar los planos hechos por Miguel Constanzó para la fábrica de las Casas Reales y cárcel de San Luis Potosí, señalaría que los arcos de las bóvedas estaban "sobre impostas al aire", lo que para el académico constituía una aberración del viejo estilo que debería remediarse.

El destino del diseño y proyecto de construcción de las Nuevas Casas Reales no estaba ya en manos del Cabildo de la ciudad de San Luis Potosí, cuya única propuesta, por ser barroca, había sido rechazada tajantemente. Al tiempo que enviaba el dictamen al Ayuntamiento potosino, el Virrey tomó la resolución de señalar al académico que se haría cargo de formar un nuevo diseño. En una carta fechada el 27 de octubre, un mes después de la enviada al Cabildo de San Luis, el Virrey dio cuenta de su decisión:

Desechados por una infinidad de defectos, hojarascas y churriguerías que se notaron en los adjuntos planos hechos para la construcción de Casas Reales y Cárcel de la ciudad de Potosí, he nombrado a vuestra merced para que por sus propias dimensiones forme desde luego otros, con arreglo a la Ilustración que en el día tiene la arquitectura, procediendo con este encargo con la brevedad más posible. Dios guarde a V. S. ms. as. México, octubre 27 de 1790. El conde de Revilla-Gigedo. (AGN, Indiferente virreinal/Obras públicas, Caja 6303, exp. 24, 1790, f. 9r)

La carta estaba dirigida al teniente coronel de Ingenieros Miguel Constanzó; se perdió así el rastro del proyecto barroco de Ureña.

\section{Sebastián Miguel Francisco Constanzó}

El ingeniero Miguel Constanzó nació en la ciudad condal de Barcelona, probablemente en el año de 1739 (Moncada Maya 131). Se sabe que se formó como ingeniero militar, que ingresó al Real Cuerpo de Ingenieros a los 23 años y que a los 
25 emprendía su viaje rumbo a Nueva España, donde realizaría lo más importante de su labor como ingeniero militar (131). Su capacidad de trabajo y disposición ante la Corona le permitieron un rápido ascenso, y se convirtió en una pieza clave en la tarea reformista borbónica que se llevaba a cabo en Nueva España.

Como ingeniero militar, Miguel Constanzó desempeñaba múltiples tareas de orden urbano, como trazar y rehabilitar caminos, conducción de agua y obras civiles, entre las cuales destacaban aquellas que influían directamente sobre aspectos defensivos - tema que apremiaba en España y sus territorios de ultramar-, como lo era el diseño de fuertes; sin embargo, fue gracias a la calidad de su trabajo como el ingeniero Constanzó se incorporó a la Academia en 1782, como profesor de matemáticas, cargo en el cual participaría en calidad de censor de la tarea constructiva, pero también como proyectista (Fuentes Rojas 32).

Resultaba difícil que un ingeniero militar participara en proyectos de arquitectura civil; y de hecho, esta era limitada. Sin embargo, existieron algunos que, por su calidad y formación, pudieron realizar con cierta frecuencia estos trabajos (Moncada Maya 118). Más aún, la participación de ingenieros militares, ya fuese en la proyección - principalmente- o en la dirección de obras civiles, tenía mucho que ver con que estas se realizaran en ciudades de interés estratégico militar (118), y no por ser obras del común, para las cuales existían alarifes calificados por la Academia o por los Ayuntamientos. Dentro de la Academia y fuera de ella, Constanzó era considerado como un excelente dibujante (196), pero más importante aún era que se lo consideraba un hombre de confianza, serio en su trabajo y su desempeño. No por otra causa, ascendió con cierta prontitud en el escalafón de los ingenieros militares ${ }^{12}(165)$. Esta confianza, aunada a sus habilidades e inteligencia en la construcción, amén de que la ciudad de San Luis era de interés estratégico militar, fueron factores que llevaron al Virrey a designarlo en 1790 como el encargado de diseñar unos planos para las Nuevas Casas de San Luis "con arreglo a la Ilustración que en el día tiene la arquitectura". Algunos autores mencionan que los planos fueron entregados hasta abril de 1796 (Montejano y Aguiñaga 1973: 48; Moncada Maya 285); sin embargo, tenemos noticia de este acontecimiento en junio de 1795 :

Contaduría de Propios: al señor Intendente D. Bruno Díaz de Salcedo. Acompaño a V.S. el expediente promovido sobre construcción de Casas Reales en esa Capital y planos levantados por el Teniente Coronel de Ingenieros D. Miguel Constanzó, a fin de que enterado V.S. de lo pedido en el asunto por

12 El 2 de octubre de 1785 recibió el nombramiento de Coronel e Ingeniero en Jefe, y en 1797, el de cuartelmaestre del Ejército de Veracruz. 
el señor fiscal de lo civil, y de mi decreto de conformidad, disponga su cumplimiento. Dios, México, 17 de junio de 1795. (AGN Real Hacienda/Bienes de Comunidad, vol. 2, exp. 268, f. 341r).

La demora obedeció, naturalmente, a las constantes obligaciones que como ingeniero militar tenía, sin contar con las revisiones de planos y proyectos que, como parte de la Academia de San Carlos, se le encargaban.

Sumado a la demora que se justifica por las múltiples ocupaciones del ingeniero, tenemos el hecho de que, como correspondía, los planos debían ser revisados por los peritos de la Academia, lo cual demoraría aún más la entrega del proyecto al Cabildo potosino. La confianza que se le tenía no lo eximía de revisiones ni le otorgaba prerrogativas. En carta fechada en mayo de 1796 - casi un año después de que tenemos noticia de la hechura de los planos-, el virrey Branciforte pidió a la Academia de San Carlos que revisara, para su aprobación, los planos ejecutados por Constanzó:

En junta superior de Propios, celebrada el día 22 del corriente, he resuelto se proceda a la construcción de las Casas Reales y cárcel de la ciudad de San Luis Potosí, previa la correspondiente aprobación o reforma de los planos que levantó el señor Coronel de Ingenieros don Miguel Constanzó, los que acompaño a V.S. con el indicado fin, esperando que concluida aquella operación me los devuelva con la posible brevedad. (AAASC, gaveta 4, expediente 581 , carta 4)

La carta y los planos entregados al director de la Academia demorarían una semana en llegar al director de arquitectura, quien era el propio Antonio González Velázquez, el cual de nueva cuenta se encargaría de revisar el proyecto para San Luis; tres meses después devolvió los planos al director de la Academia, con la siguiente anotación:

Consecuente con la orden de la Junta Superior de Gobierno que con fecha de 4 de mayo del presente año me ha remitido V. S. con los planos para fábrica de Casas Reales y cárcel de San Luis Potosí, he procedido a su reconocimiento por cumplir con la exactitud que exige la obra, y hallo en lo substancial del Edificio que en los bajos se hallan algunas oficinas de bóveda cuyos arcos existen sobre impostas del aire, lo que me parece debe remediarse formando sus pies derechos que suban a recibirlos desde el cimiento, lo que puede efectuarse al tiempo de construir la obra; y es cuanto debo decir sobre el particular Dios que a V. m. a. México, y Agosto 29 de $1796 .{ }^{13}$ (AAASC Gaveta 5, expediente $714 \mathrm{a}$, carta 1 )

13 Cfr. Montejano y Aguiñaga (1973: 48). El padre Montejano refiere el mismo dato, aunque con diferencia en la fecha y sin la recomendación emitida acerca de los arcos por el revisor Antonio González Velázquez. 
El dictamen de Velázquez es poco menos que escueto; acaso sus múltiples ocupaciones le impedían profundizar, o acaso el proyecto cumplía en lo general con lo previsto para una construcción de esta envergadura. Lo cierto es que el director de arquitectura volvió sobre sus pasos y criticó de nueva cuenta todo lo que no consideraba propio del gusto ilustrado, y así, cualquier reminiscencia barroca, como el efecto de los arcos sobre impostas voladas que presentaba en su diseño Constanzó $^{14}$, fue cuestionada, como lo hiciera años después con los arcos del tribunal de la Inquisición. La carta, con copia certificada, del dictamen del arquitecto fue devuelta al Virrey el 5 de septiembre de 1796 (AAASC gaveta 5, expediente 714a, carta 2), con lo cual, unos días después, se daría la orden de que se iniciaran los trámites necesarios — sobre todo, en materia económica— para la construcción del edificio proyectado por Constanzó. Los planos tardarían todavía tres años en pasar del papel a la piedra; sin embargo, su historia continuó a la par con la recaudación de fondos y con nuevos censores.

Algunos años después, gracias a que el proyecto de construcción estaba siendo revisado por el maestro alarife de la ciudad, José María de la Candelaria Pérez, los planos fueron tomados como referencia para determinar el presupuesto de lo que faltaba, y con esto, el esclarecimiento de cuántos y qué contenía cada uno de ellos. En una carta fechada en San Luis Potosí el 11 de diciembre de 1806, el maestro Pérez mencionó la existencia de cuatro planos para el proyecto de las Nuevas Casas Reales; el primero de ellos comprendía las asesorías en la planta inferior; el segundo mostraba las Casas Capitulares y cárcel en el plano superior; el tercero mostraba la fachada principal del edificio, y, finalmente, el cuarto plano manifestaba los alzados de las Casas Reales (AGN Intendencias, Vol. 71, s. fol.). Estos planos estaban en poder del maestro alarife y le fueron solicitados, junto con su presupuesto, para su revisión. La negativa a entregarlos, por parte del Cabildo, nos esclarece aun más la forma y el estado en que se encontraban los planos ejecutados por el ingeniero:

... se suspende por ahora la remisión [a México] de los planos formados por orden superior por el señor Coronel de Ingenieros don Miguel Constanzó (cuyo expediente debe obrar en esa superior), a causa de que estos sobre ser cuatro, están en sus correspondientes bastidores delineados [sobre] papel marquilla, puestos sobre lienzo, y por lo mismo en riesgo de [mal]tratarse y romperse, por lo que acordó este Cabildo así, si representa a V.E. bajo la protesta de que se remitirán con un propio si necesario fuere. (AGN Intendencias, Vol. 71, s. fol.)

$14 \quad$ Tampoco era la primera vez que Constanzó diseñaba cuartos cuyos arcos descansaban sobre impostas voladas: en el proyecto de las nuevas Oficinas de la Real Casa de Moneda de México de 1779 se observa esta misma solución. 
Esta misiva, firmada el 24 de abril de 1807 por José Ruiz Aguirre, dirigida al virrey don José de Iturrigaray, encontraría por respuesta, el 13 de febrero de 1808, la exigencia de que "fueran enviados [los planos] en cañón de hojalata para que, junto con los cálculos y cuentas, llegaran al señor Regente de Real Aduana" (AGN Intendencias, Vol. 71, s. fol.). La idea era que estos planos y el presupuesto fueran nuevamente revisados en la Academia de San Carlos, con el fin de ver la pertinencia tanto del presupuesto como de la continuación del diseño formado por Constanzó, en vista de los escasos fondos con que contaba el Ayuntamiento. En esta ocasión los planos serían examinados por el ilustre director de escultura de la Academia, Manuel Tolsá. El 12 de mayo de 1808 el director recibió la orden de revisar el expediente, y en diciembre emitía su dictamen:

... en cuanto a suprimir, por razón de economía o menores gastos para la conclusión de dicho Edificio, sólo podrá beneficiarse en el remate de la fachada principal, que es el cuerpo elevado donde sientan los jarrones, como expresa el plano número 1, dejando el pretil en la azotea al mismo alto que en los costados. Por lo demás no conviene suprimir nada, así por la comodidad del destino del dicho edificio, como por la sencillez y buen gusto de los planos, y más estando ya en la altura que se halla y adelantada, como manifiesta la invención de más de cincuenta mil pesos que van gastados, en un País en donde los materiales son a precios tan cómodos [...] a que se agrega a favor de la continuación de dicha obra el uso tan recomendable y objeto a que se dirige la buena disposición y aspecto noble de ella, pues basta decir de sus planos que son del S. Brigadier don Miguel Constanzó y la escrupulosa y económica invención de los caudales [...] Manuel Tolsá. (AGN Intendencias, Vol. 71, s. fol.)

Nuevamente los planos de Constanzó pasaban por buenos, aunque el tiempo transcurrido en la revisión de estos impedía el avance de la obra, por lo que el Cabildo los solicitó de nuevo, y no fueron devueltos hasta el 23 de febrero de 1809 , junto con una copia certificada de la revisión efectuada por Manuel Tolsá (AGN Intendencias, Vol. 71, s. fol.). Con esta información perdemos nuevamente de vista el destino de los planos del teniente coronel de ingenieros don Miguel Constanzó; nos queda constancia de que en lo relativo a su propuesta poco o nada se cambió, y de que, de hecho, su proyecto cumplió cabalmente con las exigencias de la Academia de San Carlos. 
Figura 1. Construcción de las Nuevas Casas Reales, $C a .1810$, sobre el diseño de Miguel Constanzó

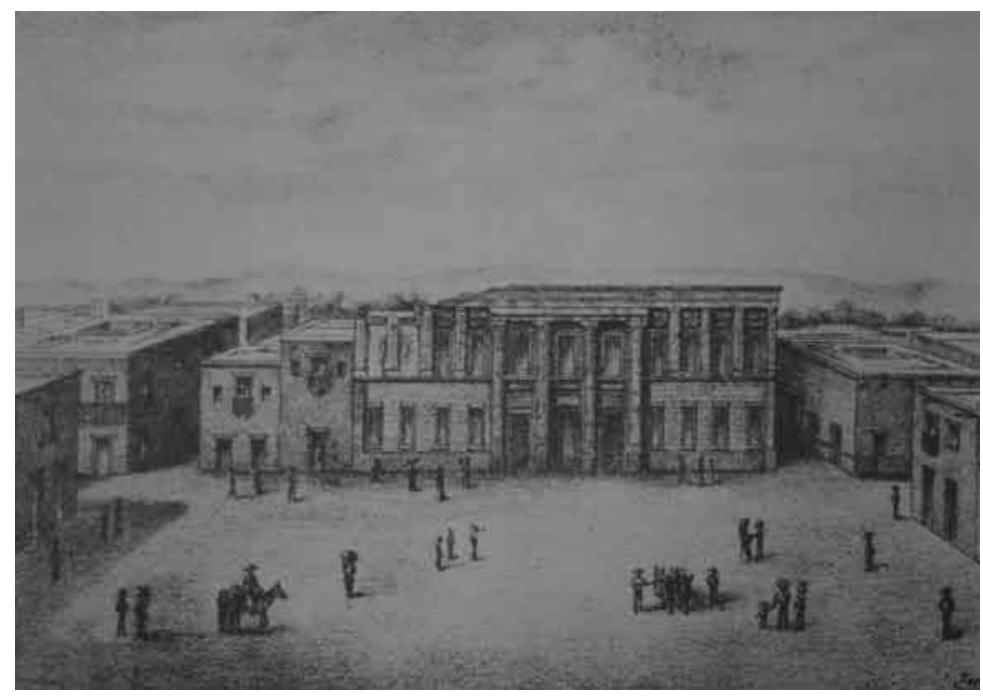

Fuente: Grabado. Nereo Rodríguez Barragán, El palacio de gobierno de San Luis Potosí. Apuntes para su historia. San Luis Potosí: Talleres Gráficos de la Editorial Universitaria, 1950.

Figura 2. Palacio de gobierno de San Luis Potosí, a finales del siglo XIX. Diseño de Miguel Constanzó

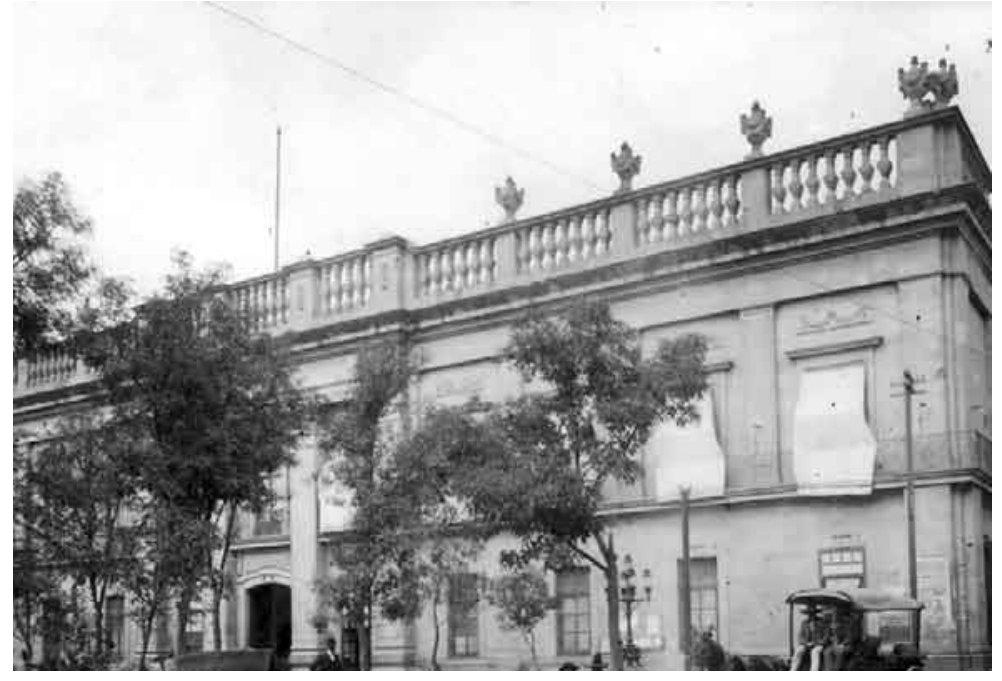

Fuente: Fotografía. Archivo fotográfico de la Biblioteca Ricardo B. Anaya. 


\section{Figura 3. Palacio de gobierno de San Luis Potosí, vista actual}

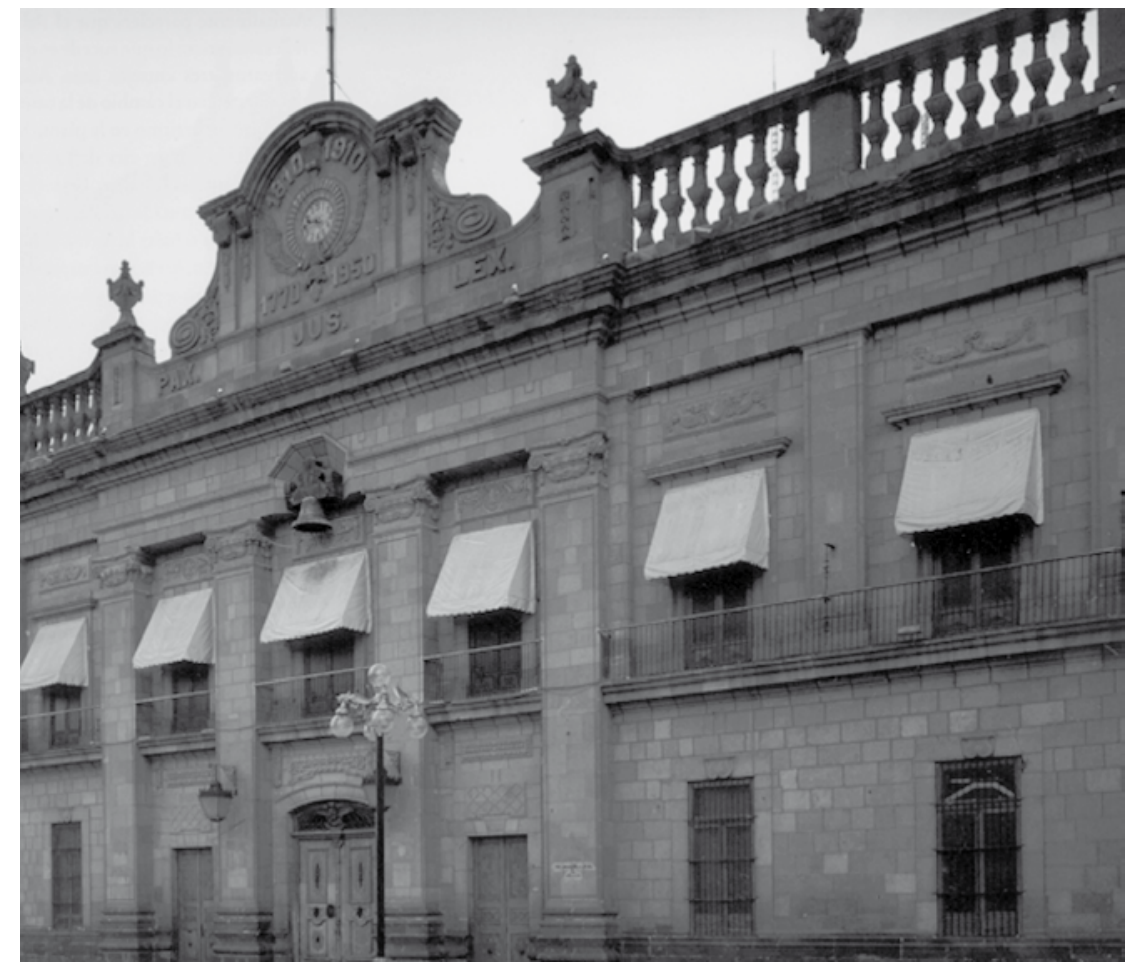

Fuente: Fotografía del autor.

\section{Conclusiones}

Al margen del destino que tuvo la construcción de las Casas Reales de San Luis Potosí, debemos considerar que detrás de este proyecto se sucedió una serie de diseños que transitaron entre períodos, donde el cambio de las mentalidades se daba de forma un tanto atropellada e iba definiendo - entre otras cosas - el rumbo de lo que sería la nueva concepción del arte y el espacio. Escenario de un conflicto constante entre personajes y entidades, este período nos permite ver a algunos defendiendo un estilo y forma de hacer que fenecía frente a la inminente llegada del progreso institucionalizado. Así, entre barroco y Academia, y acomodándose a necesidades tanto políticas como económicas y sociales, los protagonistas del diseño de las Casas Reales se dieron a la tarea de interpretar, de acuerdo con situaciones particulares, un espacio que, necesario y urgente, se vio frenado en numerosas ocasiones por ese mismo afán de progreso que caracterizaba al movimiento ilustrado. La historia de las Casas Reales de San Luis Potosí aún muestra ese carácter de transición, ese discurrir entre lo barroco y lo académico. 
El prolongado proceso que se dio entre diseños y su rechazo o aprobación nos permite vislumbrar que, a pesar de la eficiencia que el Estado buscaba, la administración y las instituciones que representaban al Estado mismo estaban llenas de burocracia y manejos poco claros, y que no obstante la conformación de las Intendencias para mejor control de los asuntos administrativos, económicos y militares, las distancias geográficas seguían influyendo de forma determinante y dilataban muchas veces los procesos. Al final, el progreso no se podía detener, y el centralismo Borbón y su mentalidad ilustrada, representados en la Academia como institución que salvaguardaba los intereses artísticos y culturales de la Corona española, terminaron por imponerse, y ello se puso de manifiesto en el resultado final; no así en el prolongado tiempo que tomó diseñar y aprobar un edificio que había sido planeado 28 años atrás. Es así que, pasando por soluciones castrenses y coqueteos barrocos, y culminando en la sencillez y rigor de la Academia, el edificio y su concepción se circunscriben en la historia como una muestra del cambio de mentalidades, testigo de la transformación que en sustancia y esencia se estaba dando en Nueva España; faltaría aún tiempo para que el rigor académico se transformara en la consolidada austeridad neoclásica. Ya con un diseño aprobado, la misma burocracia y la falta de dinero en la intendencia potosina postergarían algunos años más el inicio de la construcción que lo materializara, y este proceso se vería nuevamente interrumpido por la acometida independentista que comenzaba en los albores del siglo XIX. 
de la histaria

\section{Bibliografía}

\section{Fuentes primarias}

Archivo de la Antigua Academia de San Carlos, México (AAASC).

Archivo General de la Nación, México (AGN).

Archivo Histórico del Estado de San Luis Potosí, San Luis Potosí, México (AHESLP).

Feliciano Velázquez, Primo. "Descubrimiento y población de las Minas de Catorce (1779)". Colección de documentos para la historia de San Luis Potosí. T. 3. San Luis Potosí: Imprenta del Editor, 1898.

Tosca, Thomas Vicente. Compendio Mathematico en que se contienen todas las materias mas principales de las Ciencias, que tratan de la Cantidad. Que compuso el Doctor Thomas Vicente Tosca, Presbytero de la Congregación del Oratorio de S. Felipe Neri de Valencia. T. 5. Que comprende Arquitectura civil, Montea, y Canteria (Arquitectura militar, pirotechnia, y artilleria). De los principios o máximas de la fortificación. Madrid: Imprenta de Antonio Marín, 1712.

Yale University Library, New Haven, Estados Unidos (YUL).

\section{Fuentes secundarias}

Fuentes Rojas, Elizabeth. La Academia de San Carlos y los constructores del Neoclásico. Primer catálogo de dibujo arquitectónico (1779-1843). México: Universidad Nacional Autónoma de México, 2002.

Gálvez, José de; Castro Gutiérrez, Felipe y Croix Croix, Carlos Francisco de. Informe sobre las rebeliones populares de (1767) [Edición, índice, prólogo y notas de Felipe Castro Gutiérrez]. México: Universidad Nacional Autónoma de México, 1990.

García Salinero, Fernando. Léxico de alarifes de los siglos de oro. Madrid: Real Academia Española, 1968.

Martínez Rosales, Alfonso. El gran teatro de un pequeño mundo: El Carmen de San Luis Potosí (1732-1859). México: El Colegio de México; Universidad Autónoma de San Luis Potosí, 1985. 
Moncada Maya, José Omar. El ingeniero Miguel Constanzó: Un militar ilustrado en la Nueva España del siglo XVIII. México: Universidad Nacional Autónoma de México, 1994.

Monroy Castillo, María Isabel y Calvillo, Tomás. Breve historia de San Luis Potosí. México: Fondo de Cultura Económica, 1997.

Montejano y Aguiñaga, Rafael. El Palacio Municipal de la ciudad de San Luis Potosí. San Luis Potosí: Biblioteca de Historia Potosina, 1972.

--- El Palacio de Gobierno de San Luis Potosí. San Luis Potosí: Biblioteca de Historia Potosina, 1973.

---El Real de Minas de la Purísima Concepción de los Catorce. San Luis Potosí: Academia de Historia Potosina, 1975.

Reséndiz García, Alfonso Justino. "El taller de Felipe de Ureña en Aguascalientes y la difusión del barroco estípite en la región". Primer certamen histórico-literario. Cuento, ensayo, novela, poesía, teatro. Aguascalientes: Presidencia Municipal de Aguascalientes, Archivo Histórico de Aguascalientes, 1992.

Rodríguez G. de Ceballos, Alfonso. El Siglo XVIII. Entre tradición y Academia. Madrid: Sílex, 1992.

Rubio Mañé, Jorge Ignacio. México en el siglo XVIII. José de Gálvez Gallardo, (1720-1787) [Francisco Rodas de Cos. inv. y recop.]. México: Secretaría de Relaciones Exteriores, Embajada de México en Madrid, Comisión de Historia, Tradición, 1983.

Santos Zertuche, Francisco. Señorío, dinero y arquitectura. El Palacio de la Inquisición de México, (1571-1820). México: El Colegio de México, Universidad Nacional Autónoma de México, 2000.

Fecha de recepción: 19 de mayo de 2008

Fecha de aprobación: 25 de septiembre de 2008 